\title{
Downregulation of miR-19a inhibits the proliferation and promotes the apoptosis of osteosarcoma cells by regulating the JAK2/STAT3 pathway
}

\author{
JIANGQIANG CHEN and ZUHUI CHEN \\ Department of Traumatology, Tiantai People's Hospital, Tiantai, Zhejiang 317200, P.R. China
}

Received January 16, 2020; Accepted June 30, 2020

DOI: $10.3892 / \mathrm{ol} .2020 .12033$

\begin{abstract}
Osteosarcoma is a malignant tumor derived from the skeletal system, often occurring in bone tissues, and it is the most common malignant tumor in the skeletal system, with more than $90 \%$ of cases being highly malignant. The present study was designed to explore the regulatory effects of microRNA (miR)-19a on the proliferation and apoptosis of osteosarcoma cells, and its influence on the activation of the Janus kinase 2 (JAK2)/signal transducer and activator of transcription 3 (STAT3) signaling pathway. The expression of miR-19a in adult SaOS-2 osteosarcoma cells was downregulated via lentiviral transfection, and the cells were divided into a control group, NC-inhibitor group and miR-19a-inhibitor group. The expression of miR-19a in each group was detected via quantitative polymerase Chain reaction (qPCR). Next, the cell proliferation and apoptosis levels in each group were detected via methyl thiazolyl tetrazolium (MTT) assay and flow cytometry, respectively, and the level of reactive oxygen species (ROS) in cells was further determined. Moreover, the expression levels of apoptosis-related proteins and JAK2/STAT3 signaling pathway-related proteins were detected through western blotting. The expression level of miR-19a in the miR-19a-inhibitor group was significantly lower than that in the control group and $\mathrm{NC}$-inhibitor group $(\mathrm{P}<0.01)$. Downregulation of miR-19a significantly reduced the proliferation ability $(\mathrm{P}<0.01)$, increased the apoptosis level of SaOS-2 cells $(\mathrm{P}<0.01)$, and significantly increased the ROS level in cells $(\mathrm{P}<0.01)$. Downregulation of miR-19a also promote cleaved caspase-3/caspase-3 expression in the OS cells $(\mathrm{P}<0.01)$ and inhibited $\mathrm{Bcl}-2 / \mathrm{Bax}$ expression $(\mathrm{P}<0.01)$. Additionally, downregulation of miR-19a markedly lowered the protein expression levels of phosphorylated (p-)JAK2, p-STAT3 and myeloid cell leukemia-1 (Mcl-1) in the cells
\end{abstract}

Correspondence to: Dr Jiangqiang Chen, Department of Traumatology, Tiantai People's Hospital, 1 Kangning Zhong Lu, Shifeng, Tiantai, Zhejiang 317200, P.R. China

E-mail: rongxia19990119@163.com

Key words: miR-19a, osteosarcoma, JAK2/STAT3 signaling pathway, apoptosis
$(\mathrm{P}<0.01)$. To conclude, downregulation of miR-19a can inhibit the JAK2/STAT3 signaling pathway in SaOS-2 cells, promote the expression of apoptosis-related proteins, and increase the ROS level in cells, thereby promoting apoptosis and inhibiting cell proliferation.

\section{Introduction}

Osteosarcoma is a malignant tumor derived from the skeletal system, often occurring in bone tissues, and it is the most common malignant tumor in the skeletal system, with more than $90 \%$ of cases being highly malignant (1). Osteosarcoma is highly prevalent in children or adolescents, and frequently occurs at the sites of bone turnover and rapid bone growth, especially the metaphysis of long bone of adolescents, and the morbidity rate of osteosarcoma in males is approximately twice that in females (2). The pathogenesis of osteosarcoma is complex, and the genetic factors, environmental factors and radiative factors are all closely related to the occurrence and development of osteosarcoma $(1,2)$. Yu et al (3) studied and found that the occurrence of osteosarcoma in children may be closely associated with the mutation of genes controlling the longitudinal growth or maturation of bone in stem cells. Research evidence of Wang et al (4) confirmed that chromosomal recombination can occur in most patients with osteosarcoma, and gene mutation is an important factor influencing the occurrence and development of osteosarcoma. MicroRNAs (miRNAs/miRs) are a type of endogenous non-coding RNAs that affect the mRNA function through complementary binding to the untranslated region (UTR) of mRNAs. The correlation between miRNAs and tumors has attracted increasingly more attention from researchers (5). There is much research evidence that miRNAs are involved in the proliferation, invasion, migration and apoptosis of a variety of tumor cells. For example, Cao et al (6) found that miR-19a can significantly enhance the sensitivity of non-small cell lung cancer to chemotherapy drugs. Tan et al (7) showed that downregulation of the expression of miR-19a can obviously reduce the proliferation, invasion and migration, and promote the apoptosis of pancreatic cancer cells. The Janus kinase 2 (JAK2)/signal transducer and activator of transcription 3 (STAT3) signaling pathway is closely related to mitochondrial-mediated apoptosis. Zhang et al (8) demonstrated that inhibition of the activation of the JAK2/STAT3 
signaling pathway can effectively suppress the proliferation of lung cancer cells. At present, there are few studies on the effects of miR-19a on the JAK2/STAT3 signaling pathway and osteosarcoma cells. Therefore, the present study aimed to explore the regulatory effect of miR-19a on osteosarcoma cells through in vitro experiments, and further analyze the effects of the JAK2/STAT3 signaling pathway on the proliferation and apoptosis of osteosarcoma cells.

\section{Materials and methods}

Reagents. The reagents, kits and antibodies included: Adult SaOS-2 osteosarcoma cells (Kunming Cell Bank, Chinese Academy of Sciences, Kunming, China), RNA extraction kit (Takara), SYBR Premix Ex Taq kit and Prime Script RT reagent Kit (Takara), Dulbecco's modified Eagle's medium (DMEM) (Gibco, Rockville, MD, USA), fetal bovine serum (FBS) (Gibco; Thermo Fisher Scientific, Inc.), TRIzol (Invitrogen; Thermo Fisher Scientific, Inc.), Radioimmunoprecipitation assay (RIPA) lysis buffer (Wuhan Guge Biotech), methyl thiazolyl tetrazolium (MTT) kit (Wuhan Boster Biological Technology Co., Ltd.), apoptosis assay kit (R\&D Systems, Inc.), reactive oxygen species (ROS) assay kit (Beyotime Institute of Biotechnology), and mouse anti-human caspase-3 (cat. no. ab13847; Abcam), cleaved caspase-3 (cat. no. ab32042; Abcam), B-cell lymphoma-2 (Bcl-2) (cat. no. ab32124; Abcam), Bcl-2 associated X protein (Bax) (cat. no. ab32503; Abcam), phosphorylated (p-)JAK2 (cat. no. ab32101; Abcam), p-STAT3 (cat. no. ab76315; Abcam), JAK2 (cat. no. ab108596; Abcam), STAT3 (cat. no. ab68153; Abcam) and myeloid cell leukemia-1 (Mcl-1) (cat. no. ab32087; Abcam) antibodies. Other reagents of unspecified sources are listed in the manuscript.

Construction of osteosarcoma cell lines with low expression of $m i R-19 a$. The expression of miR-19a in the SaOS-2 osteosarcoma cell line was knocked down by GenePharma using lentiviral transfection. At $48 \mathrm{~h}$ after transfection, the successfully transfected cells were selected to extract the total RNA. The expression level of miR-19a in cells was verified via polymerase chain reaction (PCR), and the transfection efficiency was determined. The cell lines with low expression of miR-19a were selected for stable subculture as the miR-19a-inhibitor group. The blank plasmid group (NC-inhibitor group) and blank control group (Control group) were also set up.

Detection of miR-19a expression level in cells via qPCR. The cells in the logarithmic growth phase in each group were collected and centrifuged at 10,500 x g for $10 \mathrm{~min}$, the supernatant was discarded, and appropriate amount of TRIzol was added to extract the total RNA. The total RNA concentration in each group was determined using the nucleic acid-protein quantometer (NanoDrop 2000), and the optical density (OD) value was 1.8-2.0. The reverse transcription system was prepared, and the reaction conditions are as follows: $37^{\circ} \mathrm{C}$ for $15 \mathrm{~min}$ and $85^{\circ} \mathrm{C}$ for $5 \mathrm{sec}$. The total RNA was reversely transcribed into complementary deoxyribonucleic acid (cDNA), and stored for later use. Then the qPCR system was prepared in strict accordance with the instructions of kit, and the reaction conditions were as follows: $95^{\circ} \mathrm{C}$ for $30 \mathrm{sec}, 95^{\circ} \mathrm{C}$ for $5 \mathrm{sec}, 60^{\circ} \mathrm{C}$ for $35 \mathrm{sec}$ for a total of 35 cycles. With U6 as an internal reference, the relative expression level of miR-19a in each group was calculated by $2^{-\Delta \Delta \mathrm{Cq}}(9)$. The primers were synthesized by Invitrogen; Thermo Fisher Scientific, Inc., and the primer sequences are shown in Table I.

Detection of cell proliferation ability using MTT assay. The cells in the logarithmic growth phase in each group were collected, inoculated into a 96 -well plate $\left(1 \times 10^{4}\right.$ cells/well), and cultured using complete medium with $5 \% \mathrm{CO}_{2}$ under a constant temperature of $37^{\circ} \mathrm{C}$. Then $20 \mu \mathrm{l}$ of MTT was added into each well after $12,24,36,48,60$ and $72 \mathrm{~h}$ respectively, followed by culture in an incubator for another $4 \mathrm{~h}$. After the supernatant was discarded, $150 \mu \mathrm{l}$ of dimethyl sulfoxide (DMSO) (Wuxi Yangshan Biochemical Co., Ltd.) was added into each well and shaken gently. Finally, the optical density (OD) value of the cells in each group was measured at a wavelength of $450 \mathrm{~nm}$ using a microplate reader, based on which the cell proliferation level was calculated.

Detection of apoptosis level via flow cytometry. The cells in the logarithmic growth phase in each group were collected, inoculated into a 6 -well plate $\left(1 \times 10^{5}\right.$ cells/well), and cultured using complete medium with $5 \% \mathrm{CO}_{2}$ under a constant temperature of $37^{\circ} \mathrm{C}$. After $24 \mathrm{~h}$, the supernatant was discarded, and the cells were washed twice with pre-cooled phosphate-buffered saline (PBS) and centrifuged using a centrifuge at $950 \mathrm{x}$ g for $10 \mathrm{~min}$ to prepare the cell suspension. Then the cells were resuspended, centrifuged, added with fluorescence solution and incubated in the dark at room temperature for $15 \mathrm{~min}$, followed by detection of the apoptosis level via flow cytometry strictly according to the instructions of the apoptosis kit.

Determination of ROS level in each group using DCFH-DA. The ROS level in each group was determined using DCFH-DA. The cells in the logarithmic growth phase in each group were collected, inoculated into the 6 -well plate $\left(1 \times 10^{5}\right.$ cells/well), and cultured using complete medium with $5 \% \mathrm{CO}_{2}$ under a constant temperature of $37^{\circ} \mathrm{C}$. After $24 \mathrm{~h}$, the reactive oxygen species (ROS) level in each group was detected strictly according to the instructions of the ROS assay kit: An appropriate amount of DCFH-DA $(10 \mu \mathrm{M})$ was added into the 6 -well plate, and incubated in the incubator in the dark at $37^{\circ} \mathrm{C}$ for $20 \mathrm{~min}$. After the medium was discarded, the cells were washed with pre-cooled PBS and washing solution, and observed under a confocal microscope (excitation wavelength of $488 \mathrm{~nm}$, and emission wavelength of $525 \mathrm{~nm}$ ). The higher the ROS level, the higher the brightness.

Detection of protein expression level using western blotting. The cells in the logarithmic growth phase in each group were collected, inoculated into the 6 -well plate $\left(1 \times 10^{5}\right.$ cells/well), and cultured using complete medium with $5 \% \mathrm{CO}_{2}$ under a constant temperature of $37^{\circ} \mathrm{C}$. After $24 \mathrm{~h}$, the supernatant was discarded, and the cells in each group were collected for later use. The total protein was extracted from cells in each group, and the total protein concentration was determined using the bicinchoninic acid (BCA) protein quantification kit (Wuhan Boster Biological Technology Co., Ltd.). After the sodium dodecyl sulphate (SDS) $10 \%$ gel was prepared, the 
Table I. PCR primers.

Sequence

$\begin{array}{ll}\text { miR-19a } & \text { F: 5'-TCATCACGCTGTGCAAATCT-3' } \\ & \text { R: 5'-TATGGTTGTTCTGCTCTCTGTCTC-3' } \\ \text { U6 } & \text { F: 5'-ATTGGAACGATACAGAGAAGATT-3' } \\ & \text { R: 5'-GGAACGCTTCACGAATTTG-3' }\end{array}$

F, forward; R, reverse.

protein was subjected to electrophoresis, transferred $(40 \mathrm{mg})$ onto polyvinylidene fluoride (PVDF) membranes (Millipore), and sealed with freshly-prepared $5 \%$ skim milk powder for $1 \mathrm{~h}$. Then the corresponding target bands were cut, incubated with caspase-3 (dilution: 1/2,000), Bcl-2 (dilution: 1/2,000), Bax (dilution: 1/2,000), p-JAK2 (dilution: 1/2,000), p-STAT3 (dilution: 1/2,000), JAK2 (dilution: 1/1,000), STAT3 (dilution: 1/2,000), Mcl-1 (dilution: 1/1,000) and GAPDH (dilution: 1/500) antibodies at $4^{\circ} \mathrm{C}$ overnight, washed with Tris-buffered saline with Tween ${ }^{\circledR} 20$ (TBST) for 3 times, incubated again with horseradish peroxidase-conjugated secondary antibodies (dilution: 1/2,000; cat. no. ab6721; Abcam) at room temperature for $1 \mathrm{~h}$, and washed again with TBST for 3 times. Finally, bands were exposed by enhanced chemiluminescence (ECL) detection kit (Amersham Biosciences, and analyzed by ImageJ Software (version 1.38; National Institutes of Health), and the protein bands were scanned to calculate the protein expression levels in each group.

Statistical analysis. The data are expressed as mean \pm standard deviation and were analyzed using Statistical Product and Service Solutions (SPSS) 22.0 software (IBM, Corp.). One-way analysis of variance was adopted for the data in each group. The homogeneity test of variance was performed. Bonferroni's method was adopted for the pairwise comparison in the case of homogeneity of variance, while Welch's method was used in the case of heterogeneity of variance. $\mathrm{P}<0.05$ suggested that the difference was statistically significant.

\section{Results}

Construction of osteosarcoma cell lines with low expression of $m i R-19 a$. The expression of miR-19a in SaOS-2 osteosarcoma cell lines was downregulated using lentiviral transfection. After successful transfection, the expression level of miR-19a in each group was detected via qPCR. As shown in Fig. 1, the expression level of miR-19a in the miR-19a-inhibitor group was significantly lower than that in the control group and NC-inhibitor group $(\mathrm{P}<0.01)$, indicating that the transfection was successful and subsequent experiments could be performed.

Effect of miR-19a on proliferation of osteosarcoma cells. The effect of miR-19a downregulation on the proliferation of SaOS-2 cells was detected using MTT assay. As shown in Fig. 2, the cell proliferation ability in the miR-19a-inhibitor group was significantly weaker than that in the control group

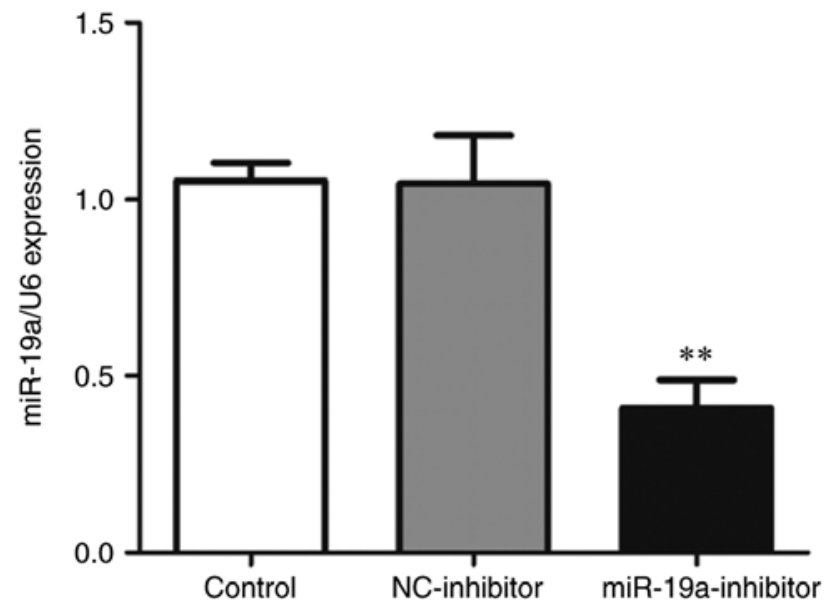

Figure 1. Expression level of miR-19a in the control, NC-inhibitor and miR-19a-inhibitor groups detected via qPCR. The expression level of miR-19a in the miR-19a-inhibitor group was significantly lower than that in the control and $\mathrm{NC}$-inhibitor groups. ${ }^{* *} \mathrm{P}<0.01$ vs. the $\mathrm{NC}$-inhibitor group. $\mathrm{NC}$, negative control

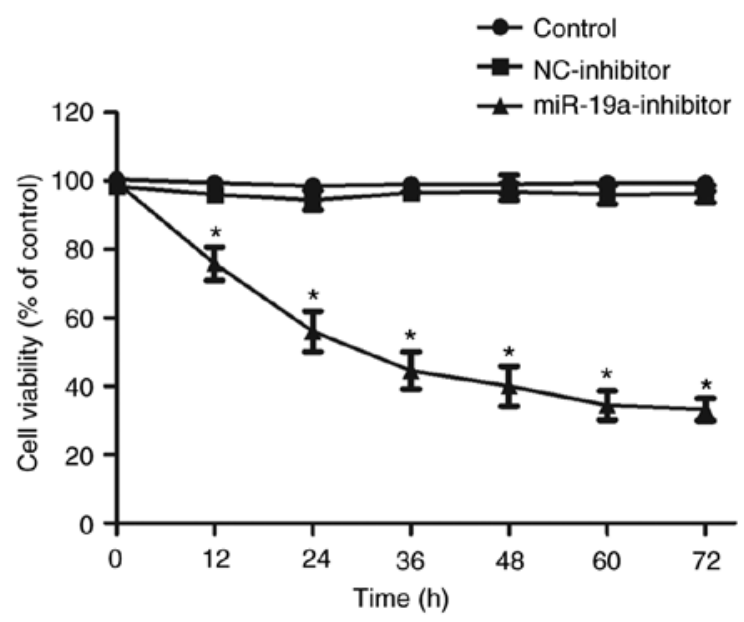

Figure 2. Cell proliferation level in the control, NC-inhibitor and miR-19a-inhibitor groups as determined using MTT assay. The cell proliferation ability in the miR-19a-inhibitor group was significantly weaker than that in the control and $\mathrm{NC}$-inhibitor groups, and the proliferation ability in the miR-19a-inhibitor group became weaker with time. ${ }^{*} \mathrm{P}<0.05$ vs. the NC-inhibitor group. NC, negative control.

and NC-inhibitor group $(\mathrm{P}<0.01)$, and the proliferation ability in the miR-19a-inhibitor group became weaker with time.

Effect of miR-19a on apoptosis of osteosarcoma cells. The apoptosis level in each group was detected through flow cytometry. The results showed that the miR-19a-inhibitor group had an obviously higher apoptosis level $(38.76 \%)$ than the control group $(4.16 \%)$ and NC-inhibitor group (13.92\%) $(\mathrm{P}<0.01)$ (Fig. 3).

Effect of miR-19a on the ROS level in osteosarcoma cells. According to the detection results of ROS level in each group using DCFH-DA, the content of ROS (60\%) in the miR-19a-inhibitor group was evidently higher than that in the control group (42\%) and NC-inhibitor group (40\%) $(\mathrm{P}<0.01)$ (Fig. 4). 
A
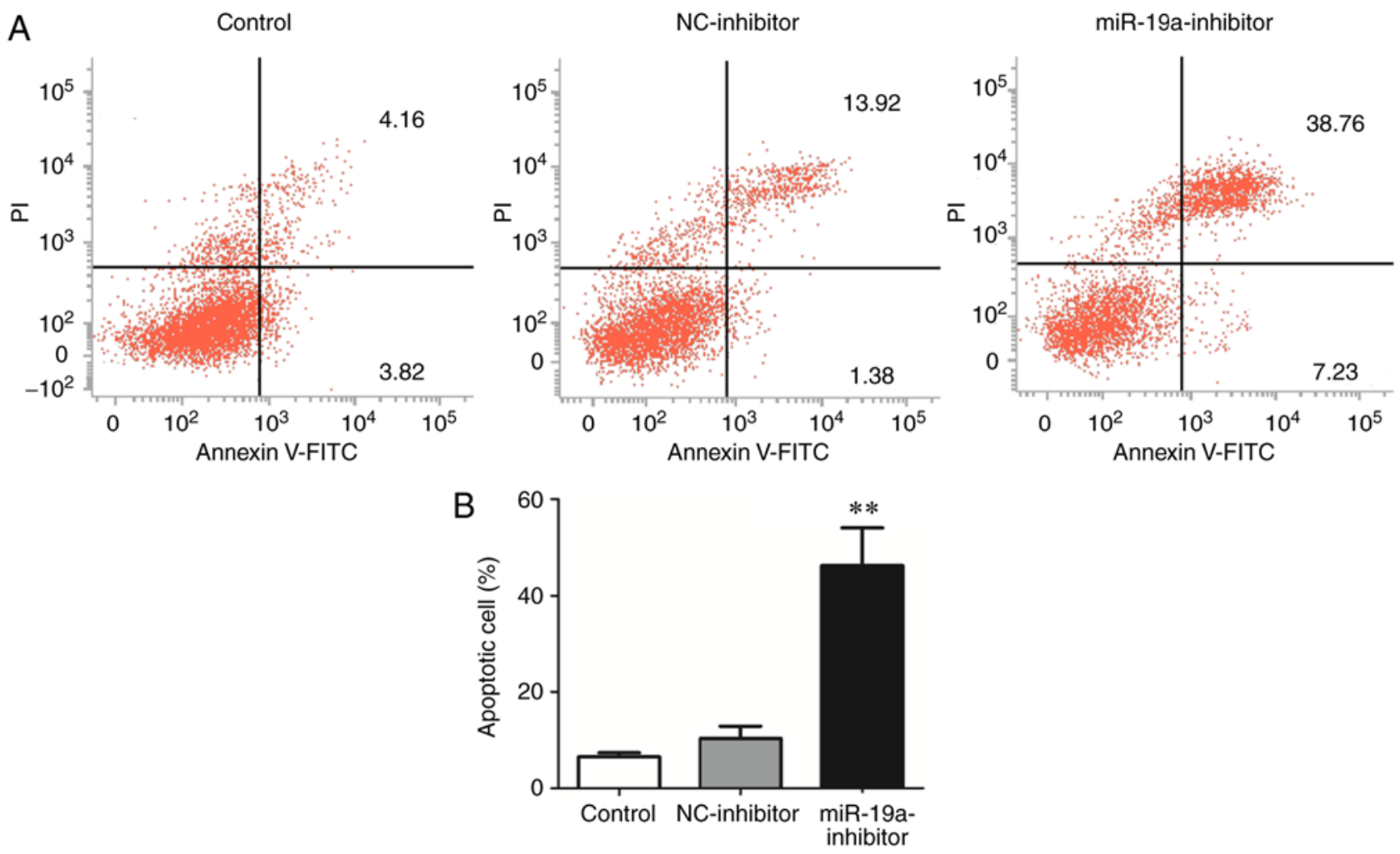

Figure 3. Apoptosis level as detected through flow cytometry. (A) Flow cytometry results and (B) statistical analysis. The miR-19a-inhibitor group had an obviously higher apoptosis level than the control and $\mathrm{NC}$-inhibitor groups. ${ }^{* *} \mathrm{P}<0.01$ vs. the NC-inhibitor group. NC, negative control.

A

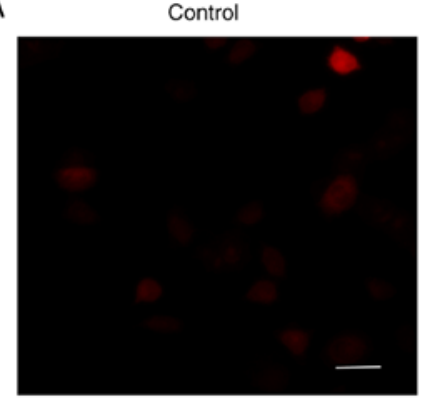

NC-inhibitor
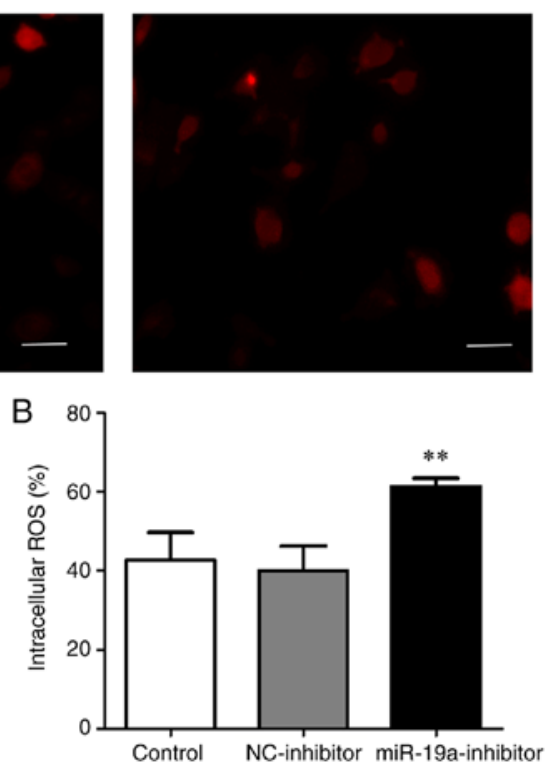

miR-19a-inhibitor

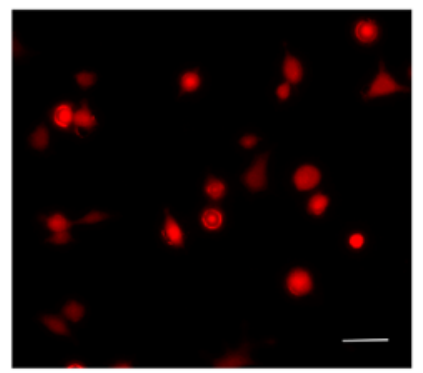

Fure 4. ROS level in the control, NC-inhibitor and miR-19a-inhibitor groups as determined by DCFH-DA. (A) Representative images of ROS levels detected by DCFH-DA. (B) Statistical analysis of the ROS levels in each group. The content of ROS in the miR-19a-inhibitor group was significantly higher than that in the control and NC-inhibitor groups. ${ }^{* *} \mathrm{P}<0.01$ vs. the NC-inhibitor group. ROS, reactive oxygen species; NC, negative control.

Effect of miR-19a on expression of apoptosis-related proteins in osteosarcoma cells. Western blotting was performed to detect the expression levels of apoptosis-related proteins in each group. The results revealed that compared with those in the control group and $\mathrm{NC}$-inhibitor group, the ratio of expression of $\mathrm{Bcl}-2 / \mathrm{Bax}$ was significantly decreased $(\mathrm{P}<0.01)$, while that of cleaved caspase-3/caspase-3 was significantly increased in the miR-19a-inhibitor group $(\mathrm{P}<0.01)$ (Fig. 5).

Effect of miR-19a on JAK2/STAT3 in osteosarcoma cells. Western blotting was also performed to detect the expression levels of JAK2/STAT3 signaling pathway-related proteins in 

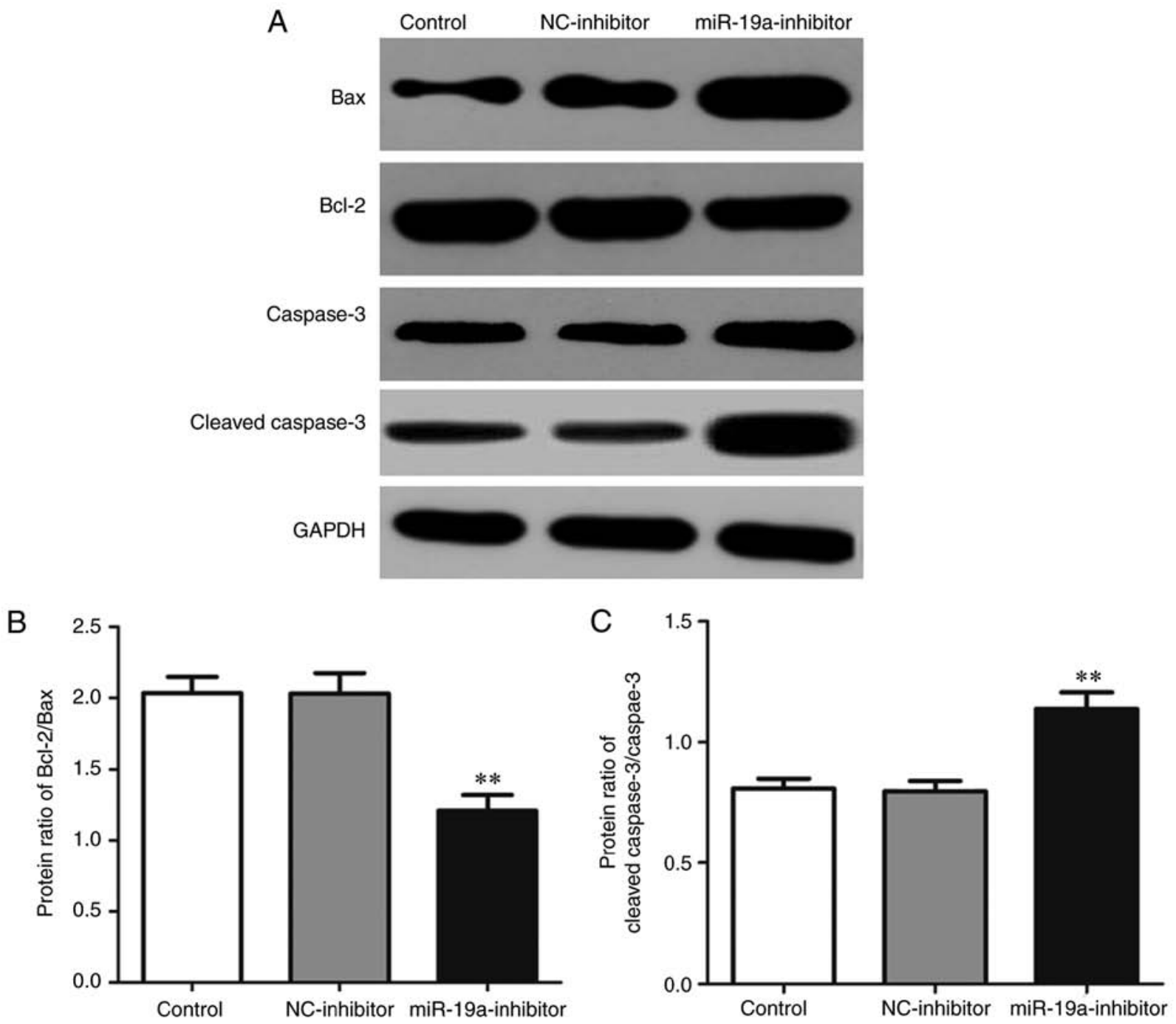

Figure 5. Expression levels of apoptosis-related proteins determined using western blotting in the control, NC-inhibitor and miR-19a-inhibitor groups. (A) Expression of apoptosis-related proteins by western blotting and statistical analyses of the ratios of (B) Bcl-2/Bax and (C) cleaved caspase-3/caspase-3. Compared with those in the control group and NC-inhibitor group, the Bcl-2/Bax ratio was significantly decreased while the ratio of cleaved caspase-3/caspase-3 was significantly higher in the miR-19a-inhibitor group. ${ }^{* *} \mathrm{P}<0.01$ vs. the $\mathrm{NC}$-inhibitor group. NC, negative control; Bcl-2, B-cell lymphoma-2; Bax, Bcl-2 associated $\mathrm{X}$ protein.

each group. It was found that the miR-19a-inhibitor group had significantly lower protein expression ratio of $\mathrm{p}-\mathrm{JAK} 2 / \mathrm{JAK}$, p-STAT3/STAT3 and Mcl-1/GAPDH than the control group and $\mathrm{NC}$-inhibitor group $(\mathrm{P}<0.01)$ (Fig. 6).

\section{Discussion}

Osteosarcoma is a malignant tumor derived from such osteocytes as fibroblasts, chondroblasts and osteoblasts, and has a high grade of malignancy. Wu et al (10) found that osteosarcoma is prone to blood, liver and lung metastases, and the incidence rate of recurrence in situ is high, for which the mechanism is closely related to gene mutation. Yin et al (11) demonstrated that miR-19a is a typical cancer-promoting gene that can facilitate the proliferation of a variety of tumor cells, and promote the invasion and migration of tumor cells through blood vessels and lymphatic vessels by downregulating the expression of target proteins and binding to vascular endothelial growth factor (VEGF) protein. Zhang et al (12) demonstrated that the expression level of miR-19a in peripheral blood of osteosarcoma patients is obviously higher than that in normal people. In the present study, it was found that downregulation of miR-19a expression in osteosarcoma cells significantly suppressed the cell proliferation ability and promoted apoptosis. Clinical evidence suggests that the expression or activation of caspase-3, a typical pro-apoptotic protein, is closely related to the occurrence and development of various tumors, and it also affects the prognosis of tumor patients (13). Bcl-2 is one of the original oncogenes that mediate apoptosis and the protein that maintains mitochondrial membrane homeostasis, which, through keeping the stability and integrity of mitochondrial membrane potential, inhibits the release of cytochrome $c$, thereby suppressing apoptosis (14). In this study, it was observed that the downregulation of miR-19a in osteosarcoma cells significantly incrased expression of caspase-3, decrease $\mathrm{Bcl}-2 / \mathrm{Bax}$ and effectively promote apoptosis.

The JAK/STAT signaling pathway is closely related to maintenance of homeostasis. As a major functional protein in the JAK family, JAK2 is able to regulate cell proliferation, 


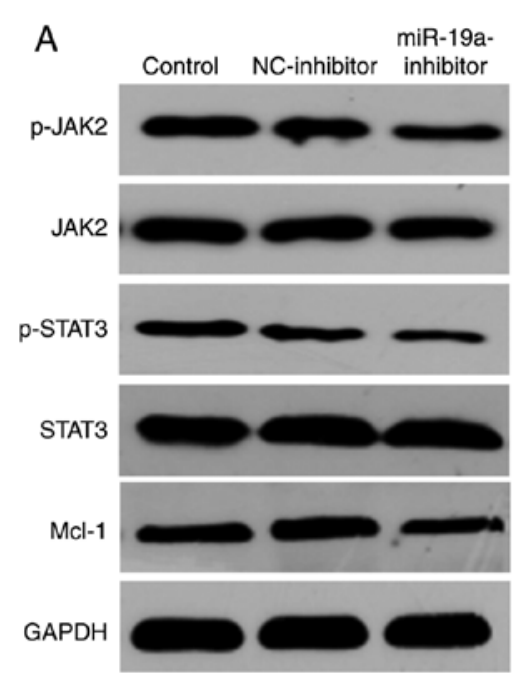

B
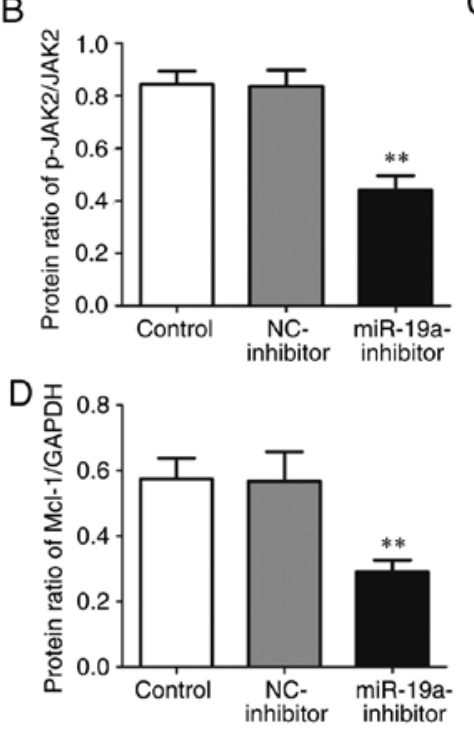

C

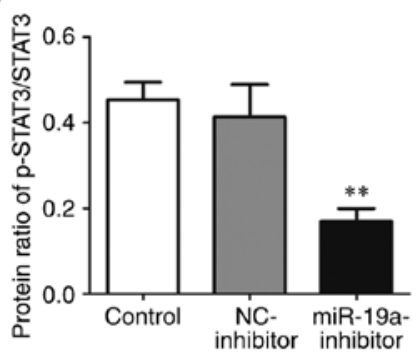

Figure 6. Expression levels of JAK2/STAT3 signaling pathway-related proteins as detected through western blotting in the control, NC-inhibitor and miR-19a-inhibitor groups. (A) Expression of JAK2/STAT3 signaling pathway-related proteins by western blotting. (B) Statistical analysis of the ratio of p-JAK2/JAK2, (C) p-STAT3/STAT3, and (D) Mcl-1. The miR-19a-inhibitor group had significantly lower protein expression ratios of p-JAK2/JAK2, p-STAT3/STAT3 and Mcl-1 than the control and NC-inhibitor groups. ${ }^{* *} \mathrm{P}<0.01$ vs. the NC-inhibitor group. NC, negative control; Mcl-1, myeloid cell leukemia-1; JAK2, Janus kinase 2; STAT3, signal transducer and activator of transcription 3; p-, phosphorylated.

differentiation, apoptosis and migration (15). The increased phosphorylation level of JAK2 protein can further activate the STAT family proteins, and then the activated STAT3 proteins remarkably upregulate the expression of anti-apoptotic proteins Bcl-2 and Mcl-1 (16). Wu et al (17) found that inhibiting the activation of the JAK2/STAT3 signaling pathway blocks the cell cycle of pancreatic cancer cells and effectively inhibits the growth, invasion and migration of tumor cells. Zhang et al (8) showed that inhibition of the JAK2/STAT3 signaling pathway can mediate the mitochondrial apoptotic pathway, thus inducing apoptosis of rectal cancer cells. A large amount of ROS produced during apoptosis is derived from mitochondria, and the increase in ROS is directly related to apoptosis (18). Moreover, according to a study of Xu et al (19), lowering the phosphorylation level of STAT3 can effectively reduce the mitochondrial membrane potential, increase the content of ROS in cells, and induce mitochondrial apoptosis. In the present study, the results revealed that downregulation of miR-19a expression in osteosarcoma cells markedly increased the content of ROS in cells, suppressed the JAK2/STAT3 signaling pathway in cells, and markedly reduced the expression levels of p-JAK2, p-STAT3 and anti-apoptotic protein Mcl-1. In addition, it was preliminarily found through flow cytometry that miR-19a significantly increased the apoptosis level of osteosarcoma cells, whose mechanism may also be to induce cell cycle arrest and downregulate the expression of cyclin. Such an uncertain mechanism is the deficiency in this study, thus further verification is needed in future research. However, there is insufficient evidence that JAK2/STAT3 is the direct target of miR-19a. The relationship of the JAK2/STAT3 signaling pathway and miR-19a and the potential target genes of miR-19a are still unknown, which will be explored in our next research stufy. In addition, only one cell line SaOS-2 was chosen for this study, and additional osteosarcoma cell lines will be employed to further investigate the effect of miR-19a.

In conclusion, the present study demonstrated that downregulation of miR-19a effectively promotes apoptosis and inhibits the proliferation of osteosarcoma cells. The related mechanism of action may be that it inhibits the JAK2/STAT3 signaling pathway, increases the ROS level in cells, activates the mitochondrial apoptotic pathway, and promotes the expression levels of apoptosis-related proteins.

\section{Acknowledgements}

Not applicable.

\section{Funding}

Not funding was received.

\section{Availability of data and materials}

All data generated or analyzed during this study are included in this published article.

\section{Authors' contributions}

JC and ZC designed the study and performed the experiments, JC collected the data, ZC analyzed the data, JC and ZC prepared the manuscript. All authors read and approved the manuscript and agree to be accountable for all aspects of the research in ensuring that the accuracy or integrity of any part of the work are appropriately investigated and resolved.

\section{Ethics approval and consent to participate}

Not applicable. 


\section{Patients consent for publication}

Not applicable.

\section{Competing interests}

The authors declare no competing interests.

\section{References}

1. Lee CM, Lee J, Nam MJ, Choi YS and Park SH: Tomentosin displays anti-carcinogenic effect in human osteosarcoma MG-63 cells via the induction of intracellular reactive oxygen species. Int J Mol Sci 20: 1508-1519, 2019.

2. Moore DD and Luu HH: Osteosarcoma. Cancer Treat Res 162 65-92, 2014

3. Yu X, Hu L, Li S, Shen J, Wang D, Xu R and Yang H: Long non-coding RNA Taurine upregulated gene 1 promotes osteosarcoma cell metastasis by mediating HIF-1 $\alpha$ via miR-143-5p. Cell Death Dis 10: 280, 2019.

4. Wang Z, Shen J, Sun W, Zhang T, Zuo D, Wang H, Wang G, $\mathrm{Xu}$ J, Yin F, Mao M, et al: Antitumor activity of Raddeanin A is mediated by Jun amino-terminal kinase activation and signal transducer and activator of transcription 3 inhibition in human osteosarcoma. Cancer Sci 110: 1746-1759, 2019.

5. Mishra S, Yadav T and Rani V: Exploring miRNA based approaches in cancer diagnostics and therapeutics. Crit Rev Oncol Hematol 98: 12-23, 2016.

6. Cao X, Lai S, Hu F, Li G, Wang G,Luo X, Fu X and Hu J: miR-19a contributes to gefitinib resistance and epithelial mesenchymal transition in non-small cell lung cancer cells by targeting c-Met. Sci Rep 7: 2939, 2017

7. Tan Y, Yin H, Zhang H, Fang J, Zheng W, Li D, Li Y, Cao W, Sun C, Liang Y, et al: Sp1-driven up-regulation of miR-19a decreases RHOB and promotes pancreatic cancer. Oncotarget 6 : 17391-17403, 2015.

8. Zhang L, Lu P, Guo X, Liu T, Luo X and Zhu YT: Inhibition of JAK2/STAT3 signaling pathway protects mice from the DDP-induced acute kidney injury in lung cancer. Inflamm Res 68: 751-760, 2019.
9. Livak KJ and Schmittgen TD: Analysis of relative gene expression data using real-time quantitative PCR and the 2(-Delta Delta C(T)) method. Methods 25: 402-408, 2001

10. Wu H, He Y, Chen H, Liu Y, Wei B, Chen G, Lin H and Lin H: LncRNA THOR increases osteosarcoma cell stemness and migration by enhancing SOX9 mRNA stability. Febs Open Bio 9: 781-790, 2019.

11. Yin Q, Wang PP, Peng R and Zhou H: MiR-19a enhances cell proliferation, migration and invasion through improving lymphangiogenesis via targeting thrombospondin-1 in colorectal cancer. Biochem Cell Biol 3: 12-27, 2019.

12. Zhang B, Liu Y and Zhang J: Silencing of miR-19a-3p enhances osteosarcoma cells chemosensitivity by elevating the expression of tumor suppressor PTEN. Oncol Lett 17: 414-421, 2019.

13. Zhang Z, Wang M, Zhou L, Feng X, Cheng J, Yu Y, Gong Y, Zhu Y, Li C, Tian L and Huang Q: Increased HMGB1 and cleaved caspase-3 stimulate the proliferation of tumor cells and are correlated with the poor prognosis in colorectal cancer. J Exp Clin Cancer Res 34: 51, 2015

14. Adams JM and Cory S: The Bcl-2 protein family: Arbiters of cell survival. Science 281: 1322-1326, 1998.

15. Groner B and von Manstein V: Jak Stat signaling and cancer: Opportunities, benefits and side effects of targeted inhibition. Mol Cell Endocrinol 451: 1-14, 2017.

16. Li S, Cui HZ, Xu CM, Sun ZW, Tang ZK and Chen HL: RUNX3 protects against acute lung injury by inhibiting the JAK2/STAT3 pathway in rats with severe acute pancreatitis. Eur Rev Med Pharmacol Sci 23: 5382-5391, 2019.

17. Wu L, Li J, Liu T, Li S, Feng J, Yu Q, Zhang J, Chen J, Zhou Y, $\mathrm{Ji}$ J, et al: Quercetin shows anti-tumor effect in hepatocellular carcinoma LM3 cells by abrogating JAK2/STAT3 signaling pathway. Cancer Med 8: 4806-4820, 2019

18. Akhtar S, Achkar IW, Siveen KS, Kuttikrishnan S, Prabhu KS Khan AQ, Ahmed EI, Sahir F, Jerobin J, Raza A, et al: Sanguinarine induces apoptosis pathway in multiple myeloma cell lines via inhibition of the JaK2/STAT3 signaling. Front Oncol 9: 285, 2019.

19. Xu Y, Zhang Q, Zhou J, Li Z, Guo J, Wang W and Wang W: Down-regulation of SOX18 inhibits laryngeal carcinoma cell proliferation, migration, and invasion through JAK2/STAT3 signaling. Biosci Rep 39: 558-569, 2019. 